OPEN ACCESS

Edited by: Alberto Di Leo,

Ospedale San Camillo, Italy

Reviewed by: Shagufta Tahir Mufti, ELMC \& H Era University, India Aditi Bhatt,

Zydus Hospitals, India

${ }^{*}$ Correspondence:

Qian Zhang

zhangqian200104@ccmu.edu.cn

${ }^{\dagger}$ These authors have contributed equally to this work and share first authorship

Specialty section: This article was submitted to Surgical Oncology, a section of the journal Frontiers in Oncology

Received: 12 May 2021 Accepted: 25 August 2021 Published: 15 September 2021

Citation:

Liang L, Han X, Zhou N, Xu H, Guo J and Zhang $Q$ (2021) Ultrasound for Preoperatively Predicting Pathology

Grade, Complete Cytoreduction Possibility, and Survival Outcomes of Pseudomyxoma Peritonei. Front. Oncol. 11:690178. doi: 10.3389/fonc.2021.690178

\section{Ultrasound for Preoperatively Predicting Pathology Grade, Complete Cytoreduction Possibility, and Survival Outcomes of Pseudomyxoma Peritonei}

\author{
Lei Liang ${ }^{1 \dagger}$, Xuedi Han ${ }^{1 \dagger}$, Nan Zhou ${ }^{1}$, Hongbin $\mathrm{Xu}^{2}$, Jun Guo ${ }^{1}$ and Qian Zhang ${ }^{3 *}$ \\ ${ }^{1}$ Department of Ultrasound, Aerospace Center Hospital, Beijing, China, 2 Department of Myxoma, Aerospace Center \\ Hospital, Beijing, China, ${ }^{3}$ Department of Gastroenterology, Beijing Friendship Hospital, Beijing, China
}

Objectives: This study aimed to investigate the value of using ultrasound (US) preoperatively for predicting pathological classification, complete cytoreduction possibility, and survival rate of patients with pseudomyxoma peritonei (PMP).

Methods: We retrospectively studied PMP patients who were scheduled for cytoreductive surgery between May 2009 and October 2019. US examination was performed before surgery. Factors related to high-grade pathology and poor completeness of cytoreduction (CC) score were identified. Associations between ultrasound characteristics and the survival status were also examined to identify independent predictive factors.

Results: PMP patients with clear ascites, abdominal lymph nodes, omental cake, abdominal mass, portal infiltration, and mesenteric involvement visible on US were considered to have high-grade pathology. Various US features were shown to be independent prognostic markers for inadequate cytoreduction in PMP patients. Portal infiltration and mesenteric involvement were significant prognostic factors for lower survival rates (hazard ratio $=3.092,3.932$, respectively). A visual nomogram including these factors was constructed to predict survival rates. The consistency index was 0.777 , which reflected relatively high accuracy. 
Conclusions: Preoperative US has the potential to predict pathological grade and resectability of PMP. Portal infiltration and mesenteric involvement were independent predictors of poor clinical outcomes in PMP patients. Furthermore, a simple-to-use nomogram derived from our study data may be a helpful visual tool in clinical practice to predict 1-, 2-, and 3-year survival rates for PMP patients.

Keywords: pseudomyxoma peritonei, ultrasound, sonography, prognosis, recurrence

\section{INTRODUCTION}

Pseudomyxoma peritonei (PMP) is a condition that is characterized by grossly disseminated intraperitoneal mucinous epithelial neoplasms and gelatinous ascites composed of copious amounts of mucin (1-3). Histopathological, immunohistochemical, and molecular-genetic data have suggested that the vast majority of PMP cases develop from mucinous carcinomas in the appendix $(4,5)$.

The recommended treatment for PMP is cytoreductive surgery (CRS) plus hyperthermic intraperitoneal chemotherapy (HIPEC) (6). This combination treatment is undoubtedly the optimal strategy associated with improved clinical outcomes. CRS aims to decrease the tumor burden in the abdominal cavity as much as possible, which is the key to treatment efficacy (7). HIPEC is delivered following CRS to clear any microscopic lesions. It is recommended that the completeness of the cytoreduction (CC) score is assessed immediately at the end of the CRS. Due to the high rate of surgical morbidity, candidate patients should be selected carefully. Lee et al. (8) reported that determining the extent of the clinical pathological classification and CC score is crucial to assessing survival outcomes. These findings should be considered when explaining to patients before surgery the benefits and risks of CRS. Thus, preoperative imaging assessment of the pathological grade and CC score is of great clinical significance.

\section{Objective}

Most PMP patients are recommended to undergo ultrasound (US) examination because of the nonspecific signs of abdominal distension and abdominal pain. The value of US is in its quantitative evaluation of PMP. This study aimed to identify preoperative ultrasonic predictors of pathological grade, resectability, and survival of PMP patients. Additionally, we sought to develop tools to determine survival time. To address this, we developed and validated a nomogram to predict mortality.

Abbreviations: PMP, pseudomyxoma peritonei; CRS, cytoreductive surgery; HIPEC, hyperthermic perioperative chemotherapy; CC, completeness of cytoreduction; US, ultrasound; CT, computed tomography; MRI, magnetic resonance imaging; HR, hazard ratio; PCI, peritoneal cancer index; ROC, receiver operating characteristic.

\section{MATERIALS AND METHODS}

\section{Study Population}

This was a prospective study of 502 PMP patients with a confirmed histological diagnosis of PMP. All patients were aged 18 years or over and underwent CRS between May 2009 and October 2019 at the Aerospace Center Hospital. US examination was conducted 2 weeks before surgery. As an optimal preoperative staging investigation, preoperative computed tomography (CT) scan was routinely performed in all patients. The study was approved by the ethics committee of the Aerospace Center Hospital. All the enrolled patients signed the informed consent. All enrolled patients were followed until February 1, 2020.

\section{Inclusion and Exclusion Criteria}

The inclusion criteria were as follows: 1) underwent CRS from May 2009 to October 2019; 2) diagnosed with PMP based on surgery or pathology; and 3) US images could be obtained in the preoperative examination 2 weeks prior to surgery at the Aerospace Center Hospital. Patients who were lost to followup were excluded.

\section{Procedure and Follow-Up}

Patients were hospitalized with a high suspicion of PMP based on imaging findings. Initially, PMP was judged and classified by ascitic fluid cytology examination. This tool is rarely applied for diagnostic purposes; however, there are currently no cytological diagnostic guidelines. Lu et al. (9) reported that mucinous epithelial and mucinous deposits in pathology cell block and smears were effective in diagnosing and classifying PMP. In cases where ascites were thick and could not be extracted, core needle biopsy of the mass and omental cake was used for diagnosis instead. The final diagnosis and classification were confirmed by postoperative pathological findings in accordance with the fourth edition of the World Health Organization (WHO) classification system $(10,11)$. The classification of low- and high-grade diseases was based on the morphologic characteristics like architecture, cytology, presence of signet ring cells, and mitotic activity (12).

Patients were scheduled for CRS at the Aerospace Center Hospital, and the quality of CRS was defined according to the Sugarbaker's CC score based on tumor distribution. No tumor or a residual tumor of less than $2.5 \mathrm{~mm}$ was scored as CC-0 (complete cytoreduction) or CC-1 (optimal cytoreduction), 
respectively. A residual tumor larger than $2.5 \mathrm{~mm}$ was scored as CC-2/3 and was considered as inadequate cytoreduction. Tolerance should be considered in the decision to undergo HIPEC treatment decision. However, only a very small number of patients refused HIPEC treatment due to personal reasons.

Six months after discharge, patients underwent blood tests and biannual imaging reexaminations. Routine follow-up involved an annual CT scan and serum tumor biomarker measurements.

\section{US Examination}

For US diagnosis, we used ultrasonic diagnostic instruments (C6-1 convex array probe, frequency 1-6 MHz; L10-2 linear array probe, frequency $2-10 \mathrm{MHz}$ ) (Supersonic Imagine, France; SAMSUNG, South Korea; Philips, Netherlands). The entire abdomen was scanned using C6-1 and L10-2 probes. The location, size, extent, border, and echogenicity were recorded if ascites, omental cake, liver scalloping, celiac, pelvic masses, or enlarged lymph nodes were present. Ascites were classified into three subtypes according to appearance: clear ascites, which were relatively anechoic, and no septum could be found in the area by US; mucus granules ascites, which showed multiple punctate high-level echoes in echo-free ascites that moved under the pressure of the transducer; and jelly-like ascites, which were gelatinous ascites with a positive fluctuation test. To assess the presence of particulates or the mobility of ascites, posture adjustment and the probe pressing manner were applied. The adjacent viscera were also scanned to exclude organ invasion. The sonographic images were reviewed by two doctors with expertise in PMP imaging with extensive 10 years of experience, who were blinded to patient data.

\section{Statistical Analysis}

Continuous variables were described as median and interquartile range (IQR). Categorical variables were described as frequencies and percentages. Univariate and multivariate logistic regression models were used to identify the factors related to high-grade pathology and high CC score. The receiver operating characteristic (ROC) curves, sensitivities, specificities, and 95\% confidence intervals (CIs) were calculated to evaluate the diagnostic performance using MedCalc statistical software (MedCalc Software Ltd., Belgium). The survival curves were plotted using a Kaplan-Meier method in GraphPad Prism (GraphPad Software, USA), and a log-rank test was used for statistical analysis. The associations between US characteristics and the survival status were examined using univariate and multivariate backward Cox proportional hazard regression models. All statistical tests were two-sided, and a significance level of 0.05 was used. Data analyses were performed using SAS software (SAS Institute Inc., USA).

A multivariate Cox regression model was used for the development of the nomogram. A simple-to-use nomogram provided a convenient graphical representation of the prediction model of survival probability that is useful for patient evaluation. Each independent variable was scored according to its contribution to the outcome. The scores of these variables were summed to provide a prediction of survival rates.

\section{RESULTS}

\section{The Demographic, Clinical, and US Characteristics of Enrolled Patients}

The median age of the 502 enrolled patients was 58 years with an IQR of 49 to 64 years. The majority of patients (63.15\%) were female. Among the PMP patients, $35.06 \%$ had no ascites under US examination, whereas $22.51 \%$ had clear ascites, $14.54 \%$ had mucus granule ascites (Figures 1A, E), and $27.89 \%$ had jelly-like ascites. Liver scalloping was observed in $73.90 \%$ of patients (Figures 1B, F), 47.01\% of patients showed significant omental cake (Figures 1C, G), $41.63 \%$ showed the abdominal mass (Figures 1D, H), and $61.75 \%$ showed involvement of lesser omental bursa. Most patients had no abdominal lymph nodes (98.21\%) (Table 1). Portal infiltration (Figures 2A, C) occurred in $58.37 \%$ of patients, and mesenteric involvement (Figures 2B, D) was observed in $17.33 \%$ of patients. These characteristics of PMP could also be visualized on CT scans. The detection ability of preoperative US and preoperative CT for PMP was compared, as Table 2 depicts. Compared with CT, US had higher accuracy in displaying ascites ( $p$ $<0.001)$, liver scalloping $(p<0.001)$, omental cake $(p<0.001)$, and lesions in lesser omental bursa $(p<0.001)$ but lower accuracy than CT for identifying portal infiltration and mesenteric involvement. Furthermore, US had an advantage in distinguishing mucus granules ascites from mucus over CT $(p<0.001)$.

\section{US Characteristics Related to Pathological Grade and CC Score}

Age and gender showed no significant relation to pathological grade. The US characteristics that were significantly associated with pathological grade were ascites $(p<0.001)$, abdominal lymph nodes $(p=0.022)$, omental cake $(p<0.001)$, abdominal mass $(p=0.007)$, portal infiltration $(p=0.008)$, and mesenteric involvement $(p<0.001)$ (Table 3). Moreover, we identified a strong correlation between CC score and presence of clear ascites $(p=0.004)$, liver scalloping $(p=0.005)$, abdominal mass $(p=$ $0.022)$, portal infiltration $(p<0.001)$, mesenteric involvement $(p<$ $0.001)$, and lesions in the lesser omental bursa $(p=$ 0.003) (Table 4).

The ROC curve of portal infiltration in relation to pathological grade showed a sensitivity of $66.0 \%(95 \% \mathrm{CI}$ : $57.9 \%-73.5 \%$ ) and a specificity of $45.0 \%$ (95\% CI: $39.7 \%-$ $50.4 \%$ ) (Figure 3A). The ROC curve of portal infiltration in relation to the CC score showed a sensitivity of $90.4 \%(95 \% \mathrm{CI}$ : $86.1 \%-93.8 \%)$ and a specificity of $73.7 \%$ (95\% CI: $67.8 \%-79.0 \%)$ (Figure 3B). Mesenteric involvement was difficult to detect in some cases but represented high specificity in predicting the CC score and survival outcomes once detected by the US. The ROC curve of mesenteric involvement in relation to pathological grade showed a sensitivity of $28.8 \%$ (95\% CI: $21.7 \%-36.6 \%)$ and a specificity of $87.7 \%$ (95\% CI: $83.8 \%-90.9 \%$ ) (Figure 3C). 


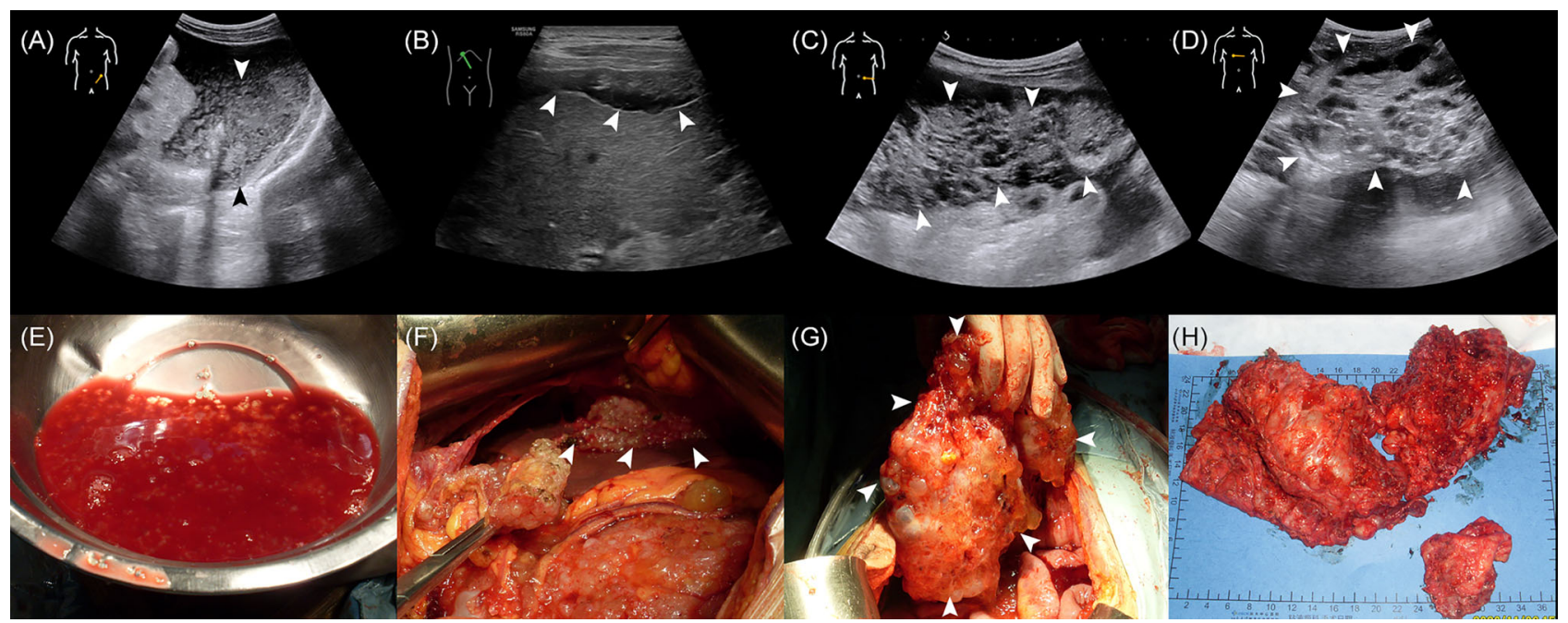

FIGURE 1 | The US characteristics and the corresponding gross specimens of PMP patients. (A, E) The absence of mucus granules ascites. (B, F) Liver scalloping. (C, G) Omental cake. (D, H) Abdominal mass.

TABLE 1 | Demographic, clinical, and US characteristics of patients.

\begin{tabular}{|c|c|}
\hline Characteristic & Median (IQR)/N (\%) total = 502 \\
\hline Age, years & $58(49-64)$ \\
\hline \multicolumn{2}{|l|}{ Gender } \\
\hline Male & $185(36.85)$ \\
\hline Female & 317 (63.15) \\
\hline \multicolumn{2}{|l|}{ Astices } \\
\hline No & $176(35.06)$ \\
\hline Clear & $113(22.51)$ \\
\hline Mucus granules & $73(14.54)$ \\
\hline Jelly-like & $140(27.89)$ \\
\hline \multicolumn{2}{|l|}{ Liver scalloping } \\
\hline No & $131(26.10)$ \\
\hline Yes & $371(73.90)$ \\
\hline \multicolumn{2}{|c|}{ Abdominal lymph nodes } \\
\hline No & $493(98.21)$ \\
\hline Yes & $9(1.79)$ \\
\hline \multicolumn{2}{|l|}{ Omental cake } \\
\hline No & $266(52.99)$ \\
\hline Yes & $236(47.01)$ \\
\hline \multicolumn{2}{|l|}{ Abdominal mass } \\
\hline No & $293(58.37)$ \\
\hline Yes & 209 (41.63) \\
\hline \multicolumn{2}{|l|}{ Portal infiltration } \\
\hline No & 209 (41.63) \\
\hline Yes & $293(58.37)$ \\
\hline \multicolumn{2}{|c|}{ Mesenteric involvement } \\
\hline No & 415 (82.67) \\
\hline Yes & 87 (17.33) \\
\hline \multicolumn{2}{|c|}{ Lesions in lesser omental bursa } \\
\hline No & $192(38.25)$ \\
\hline Yes & $310(61.75)$ \\
\hline \multicolumn{2}{|l|}{ Intraoperative $\mathrm{PCl}$} \\
\hline $\mathrm{PCl}<10$ & $104(20.72)$ \\
\hline $10 \leq \mathrm{PCl} \leq 20$ & $68(13.55)$ \\
\hline $\mathrm{PCl}>20$ & $330(65.74)$ \\
\hline CC score & \\
\hline
\end{tabular}

(Continued)
TABLE 1 | Continued

\begin{tabular}{lc}
\hline Characteristic & Median (IQR)/N (\%) total $\mathbf{= 5 0 2}$ \\
\hline CC-0/1 & $251(50.00)$ \\
CC-2/3 & $251(50.00)$ \\
HIPEC & \\
Yes & $436(86.85)$ \\
No & $66(13.15)$ \\
Origin & \\
Appendix & $479(95.42)$ \\
Others & $23(4.58)$ \\
Pathology & \\
Low-grade & $349(69.52)$ \\
High-grade & $153(30.48)$
\end{tabular}

Mesenteric involvement in relation to CC score showed a sensitivity of $31.5 \%$ (95\% CI: $25.8 \%-37.6 \%)$ and a specificity of 96.8\% (95\% CI: 93.8\%-98.6\%) (Figure 3D).

\section{US-Documented Portal Infiltration and Mesenteric Involvement as Survival Risk Factors}

The relationship between US characteristics and the outcomes of PMP patients was evaluated in the multivariate Cox proportional hazard regression model. In addition, the pathological grade, the resectability of treatment (CC score), intraoperative peritoneal cancer index (PCI), and tumor origin were investigated. In contrast to portal infiltration $(p<0.001)$ and mesenteric involvement $(p<0.001)$, several other US characteristics showed no significant relationship with the survival rates, as shown in Table 4. The log-rank test of survival rate showed that patients with portal infiltration or mesenteric involvement had poorer survival rates $\left(\chi^{2}=46.488,146.888, p<0.001\right)$ than patients with no portal infiltration or mesenteric involvement 


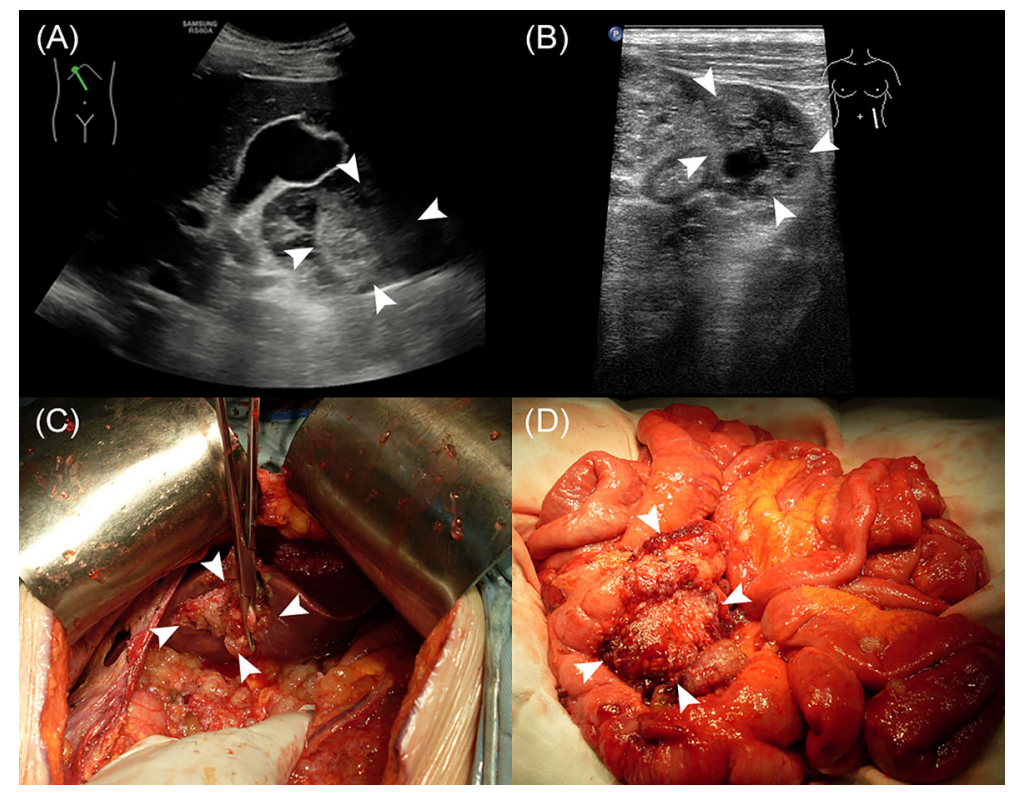

FIGURE 2 | The US characteristics and the corresponding gross specimens of PMP patients. (A, C) Portal infiltration. (B, D) Mesenteric involvement.

TABLE 2 | The diagnostic accuracy of US and CT with surgery as the gold standard.

\begin{tabular}{lccc}
\hline & CT & US & p \\
\hline Ascites & $82.67 \%$ & $96.02 \%$ & $<0.001$ \\
Ascites granules & $57.57 \%$ & $98.80 \%$ & $<0.001$ \\
Liver scalloping & $89.44 \%$ & $96.22 \%$ & $<0.001$ \\
Abdominal lymph nodes & $85.06 \%$ & $76.89 \%$ & 0.003 \\
Omental cake & $69.92 \%$ & $89.04 \%$ & $<0.001$ \\
Abdominal mass & $57.57 \%$ & $93.23 \%$ & $<0.001$ \\
Portal infiltration & $80.88 \%$ & $69.92 \%$ & $<0.001$ \\
Mesenteric involvement & $86.65 \%$ & $57.37 \%$ & $<0.001$ \\
Lesions in lesser omental bursa & $65.14 \%$ & $85.26 \%$ & $<0.001$ \\
\hline
\end{tabular}

(Figure 4). The results indicated that portal infiltration or mesenteric involvement was associated with poor prognosis (hazard ratio $[\mathrm{HR}]=3.092,[95 \% \mathrm{CI}: 1.795-5.328], \mathrm{HR}=$ 3.932, [95\% CI: 2.646-5.844]). The absence of HIPEC $(p<$ $0.001)$ and high-grade pathology $(p<0.001)$ were both independent predictors of poor survival rates. Patients who did not receive HIPEC and those with high-grade pathology had a shorter duration of survival, with an HR of 2.677 (95\% CI: 1.7943.993 ) and 2.529 (95\% CI: (1.758-3.636), respectively. There were no significant correlation between the intraoperative PCI, CC score, tumor origin, and survival outcomes in the multivariate analysis $(p>0.05)$.

A nomogram was developed using a multivariate Cox regression model to predict the 1-year, 2-year, and 3-year survival rates, helping predict the survival probability in a visual format (Figure 5). Pathological grade, HIPEC, portal infiltration, and mesenteric involvement were included in the nomogram because the Cox regression model predicted these factors as independent covariates for the risk of death. A patient's probability of individual survival was calculated by adding the scores of these selected variables. The consistency index for the nomogram was 0.777 , which reflected a relatively high accuracy in predicting the survival probability. Furthermore, the similarities between the nomogram's predicted survival rates and the actual survival rates were evaluated in 1-, 2-, and 3year validation plots. The bootstrapped resample (500 iterations) was used to verify the accuracy of the nomogram. These plots showed that the predicted survival rates closely corresponded with the actual survival rates represented by the dotted lines (Figure 6).

\section{DISCUSSION}

\section{The Utility of Preoperative US}

PMP develops from mucinous carcinomas that mostly arise from the appendix and/or ovary. According to the appearance of the ascites, the proportion of mucinous substances can be classified as clear, mucinous particles or jelly-like ascites. As a common imaging method, CT is routinely used for the preoperative evaluation of PMP patients (13). However, the small peritoneal tumors in the ascites or small septa in a mass may be indistinguishable, resulting in a decrease in diagnostic. Furthermore, the extent of disease assessed by CT consistently underestimates peritoneal spread in PMP patients due to the partial volume effect and the limited resolution of soft tissue. US is the first imaging modality in most hospitals for investigating unclear abdominal problems and is recommended for PMP patients with primary symptoms of abdominal distension and 
TABLE 3 | The univariate and multivariate logistic regression for pathology.

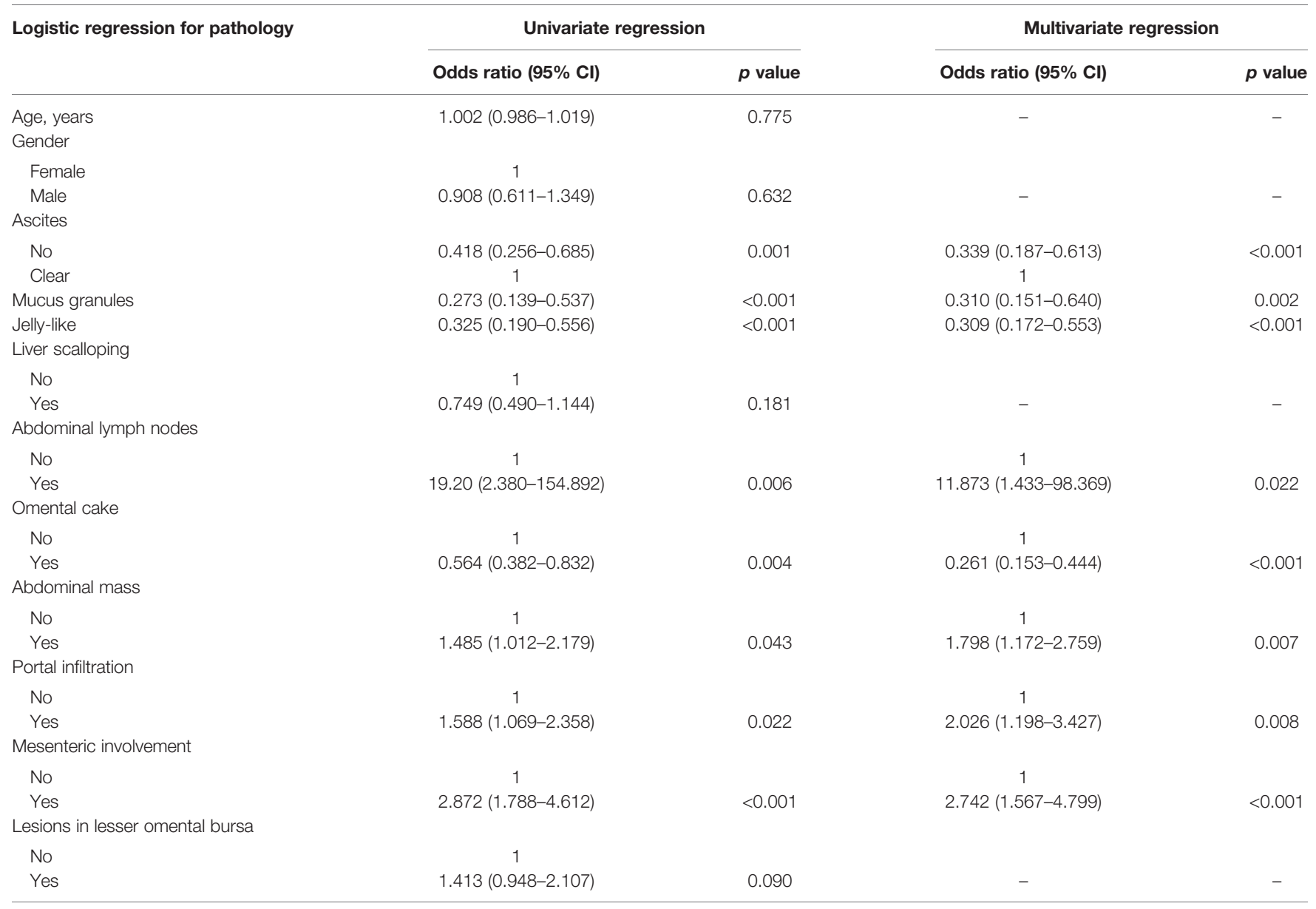

TABLE 4 | The univariate and multivariate logistic regression for CC score.

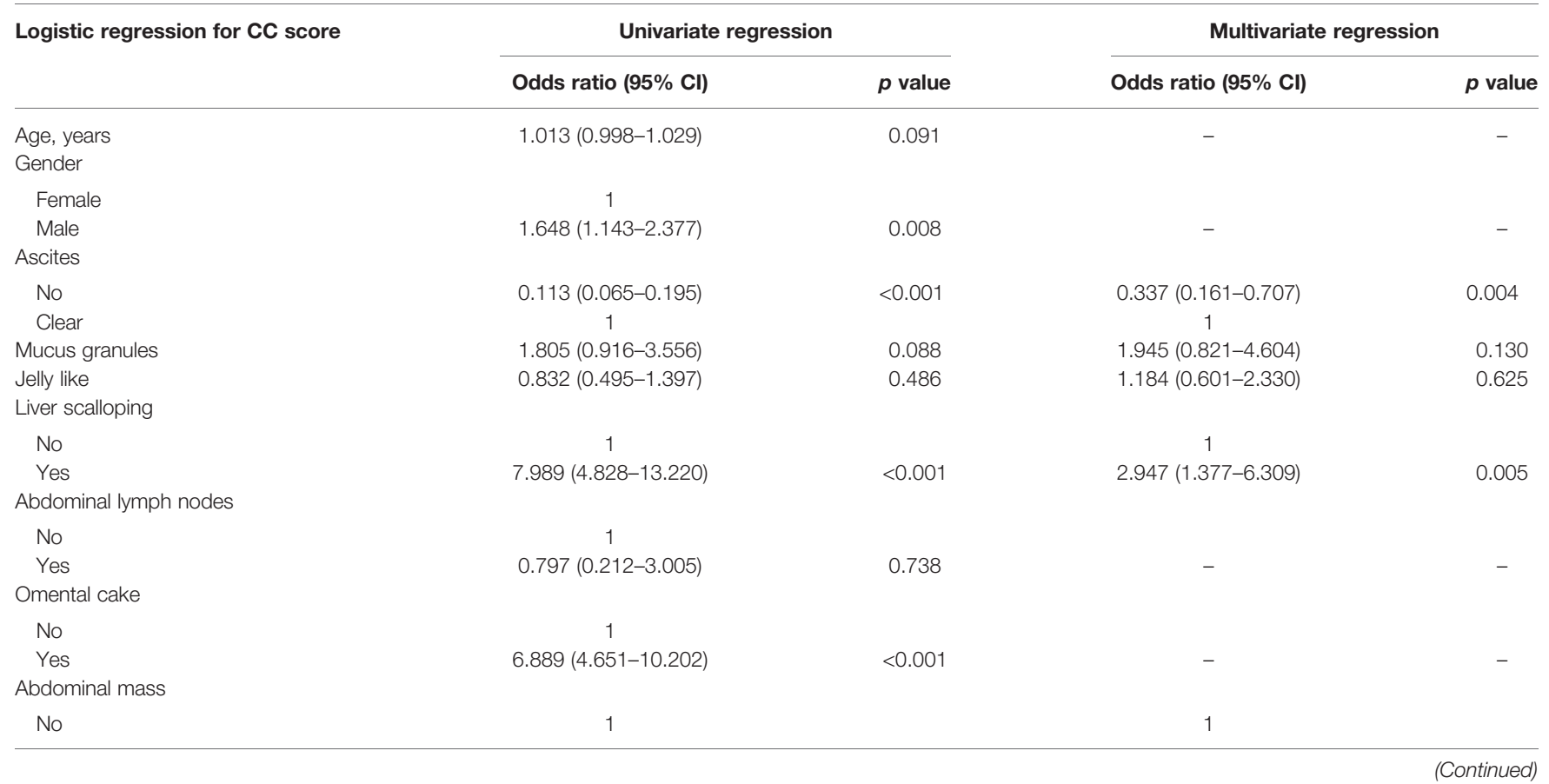


TABLE 4 | Continued

Logistic regression for $\mathrm{CC}$ score

Yes
Portal infiltration
No
Yes
Mesenteric involvement
No
Yes
Lesions in lesser omental bursa
No
Yes

\begin{tabular}{c} 
Univariate regressio \\
\hline Odds ratio $(95 \% \mathbf{~ C l})$ \\
$0.685(0.480-0.979)$ \\
1 \\
$26.512(15.985-43.970)$ \\
1 \\
$13.951(6.571-29.622)$ \\
1
\end{tabular}

15.917 (9.881-25.642)

$\boldsymbol{p}$ value
0.038

0.038

$<0.001$

$<0.001$

$<0.001$
Multivariate regression

\begin{tabular}{ll}
\hline Odds ratio $(\mathbf{9 5} \% \mathrm{Cl})$ & $\boldsymbol{p}$ value \\
\hline $0.541(0.320-0.916)$ & 0.022
\end{tabular}

0.541 (0.320-0.916)

1

$8.806(4.782-16.216)$

$<0.001$

1

7.570 (2.932-19.543)

$<0.001$

1

2.624 (1.380-4.988)

0.003
A

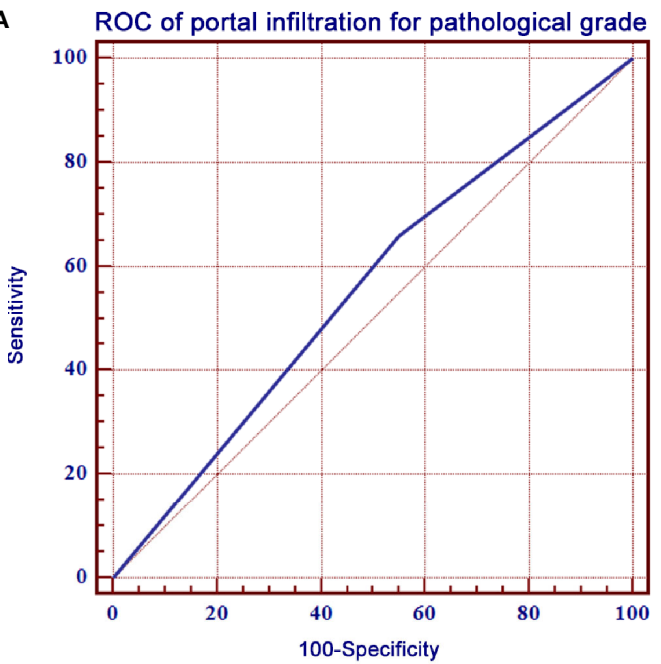

C ROC of mesenteric involvement for pathological grade

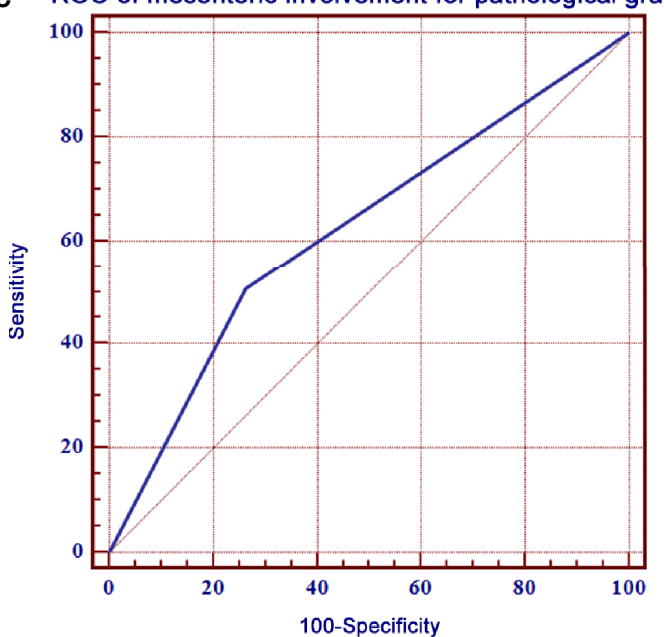

B

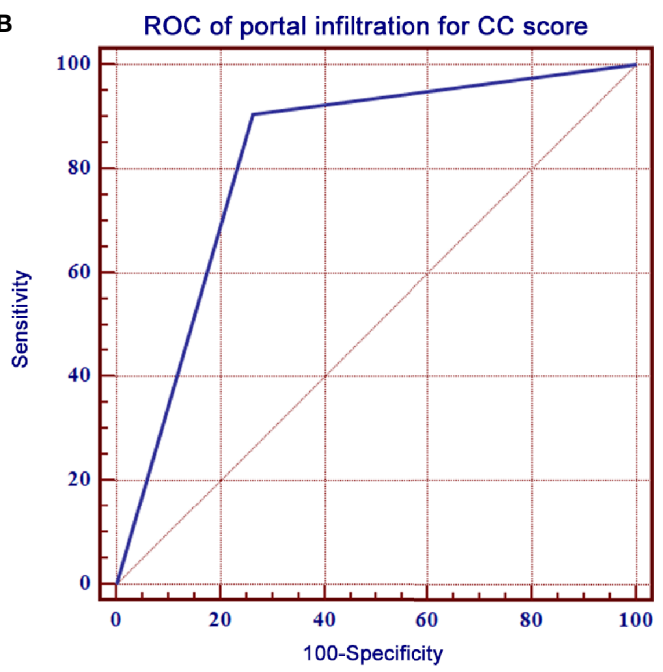

D

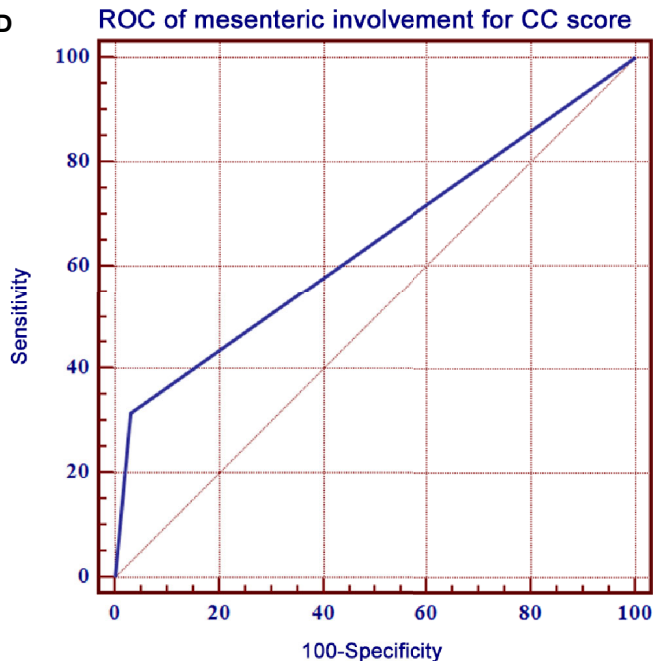

FIGURE 3 | ROC curves of portal infiltration, mesenteric involvement for pathological grade, and CC score. (A) ROC curve of portal infiltration showed a sensitivity of $66.0 \%$ and a specificity of $45.0 \%$ for pathological grade. (B) ROC curve of portal infiltration had a sensitivity of $90.4 \%$ and a specificity of $73.7 \%$ for the CC score. (C) ROC curve of mesenteric involvement showed a sensitivity of $66.0 \%$ and a specificity of $45.0 \%$ for pathological grade. (D) ROC curve of mesenteric involvement had a sensitivity of $90.4 \%$ and a specificity of $73.7 \%$ for CC score. 


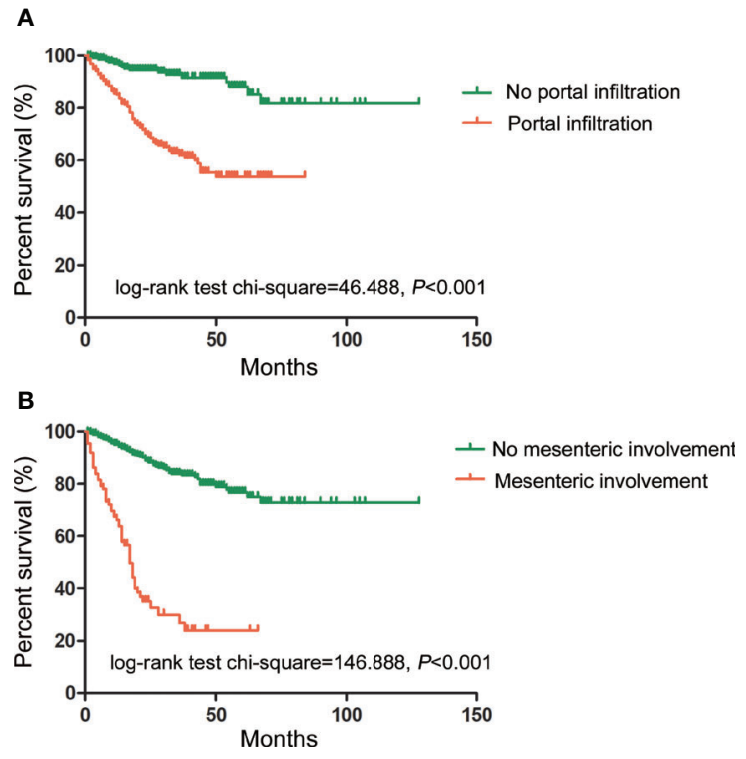

FIGURE 4 | Prognostic impact of portal infiltration and mesenteric involvement as detected by US on survival outcomes. (A) The log-rank test of survival showed that patients with portal infiltration had lower survival duration (in months) than patients with no portal infiltration. $\left(\chi^{2}=46.488, p<0.001\right)$. (B) The log-rank test of survival showed that patients with mesenteric involvement also had lower survival duration than those without mesenteric involvement. $\left(\chi^{2}=146.888, p<0.001\right)$.

pain. As an imaging technique, US is superior to CT because of its low cost, availability, and tolerability. However, the use of US in PMP patients has not been sufficiently investigated.

Several studies (14-16) have confirmed the feasibility and advantages of using US in the quantitative evaluation of PMP. Our findings showed that US provides an excellent visualization of the PMP lesions. The diagnostic accuracy of US and CT was compared to the gold standard of surgery (Table 5).
The fundamental physics underlying US lies in the difference in acoustic impedance within tissues. This offers significant advantages in observing the distribution of the lesions and allows the boundary between the ascites and the organs to be observed clearly. Furthermore, the boundary of the mucinous abdominal mass can be clearly detected. Predicting the distribution of disease accurately before surgery is valuable and is beneficial for performing a successful operation. However, due to the increased abdominal girth of PMP patients, deep exploration depth, and the imaging of intestinal gas of PMP patients, detecting mesenteric involvement by US was shown to have low sensitivity. Nevertheless, high specificity could be achieved once mesenteric involvement was detected by US.

\section{Diagnostic Value of US in Pathology}

Our results suggested that preoperative US contributed to the prediction of the pathological grade of PMP. Compared with clear ascites, mucinous particles in ascites and jelly-like ascites were more suggestive of a low-grade subgroup. A recent study (17) also showed that the mucus content of the high-grade subgroup was lower than that of the low-grade group, which was attributed to pathological and cytological differences. In the low-grade subgroup, the appendiceal neoplasm epithelial cells were positioned in a single row and the mucin secretion was typically exuberant, whereas the cells in the high-grade group were belt-shaped, island, or cribriform-shaped with severe dysplasia. The secretory function of the columnar cells was severely damaged, which led to a decrease in mucus secretion $(2,6,18)$. Moreover, the concentrated tumor mass resulted in liver scalloping and omental cake $(19,20)$. The current study results also demonstrated that the presence of omental cake predicted a higher pathological grade.

A previous investigation revealed that enlarged lymph nodes can sometimes exist in peritoneal mucinous carcinomas (21). The lesions were extensively distributed throughout the abdominal cavity in PMP patients; however, hematogenous or lymphatic metastasis rarely occurred $(22,23)$. Although rare, we

Points
Pathological grade
HIPEC
Portal infiltration
Mesenteric involvement
Total Points
1-Year Survival
2-Year Survival
3-Year Survival

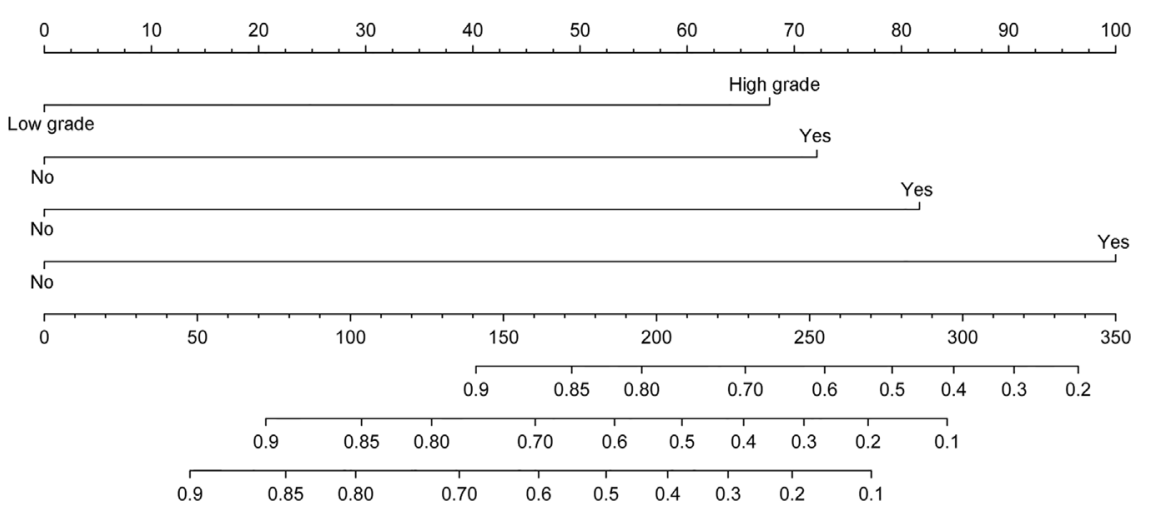

FIGURE 5 | A nomogram to predict the 1-, 2-, and 3-year survival rates in PMP patients. It is used by drawing a line perpendicular from the corresponding axis of each risk factor until it reaches the top line labeled "Points." Then, the number of points for all risk factors is summed, and a line is drawn that descends from the axis labeled "Total points" until it intercepts each of the survival axes to determine the 1-, 2-, and 3-year survival probabilities. 

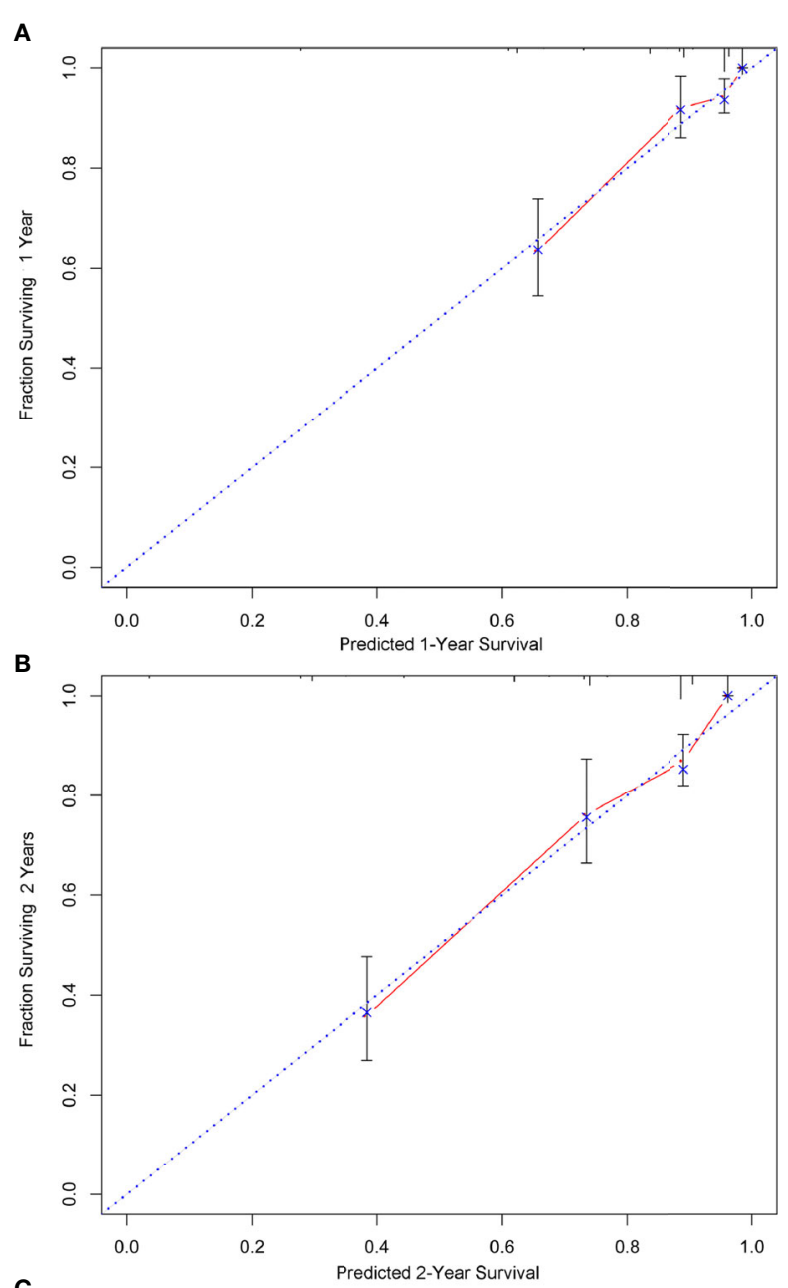

C

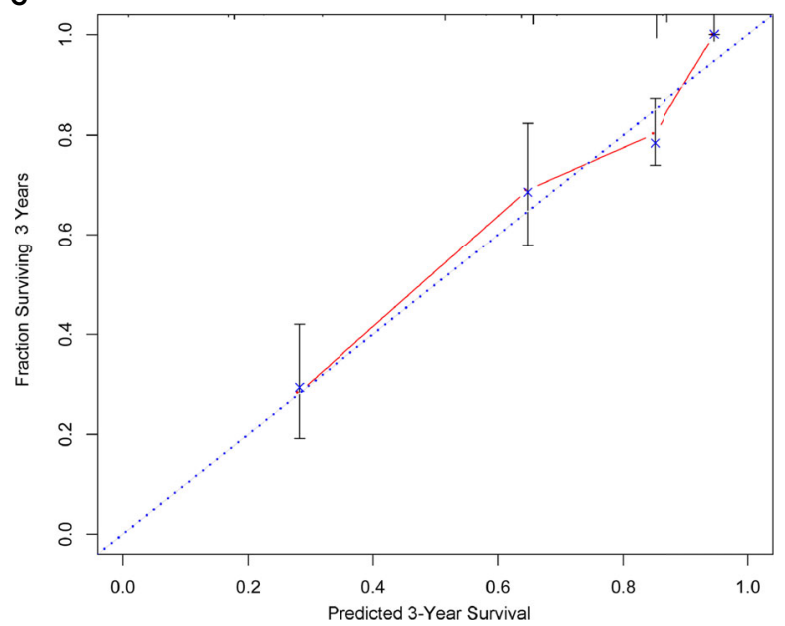

FIGURE 6 | The calibration of the nomograms for predicting 1-, 2-, and 3year survival probabilities. (A-C) show the nomograms of 1-, 2-, and 3-year survival probabilities, respectively. Plots show that the predicted survival rates closely corresponded with the actual survival rates, which are represented by the dotted lines. detected lesions with lymph node involvement that occurred in nine high-grade cases in this study. Lymph node metastasis was correlated with high-grade pathology according to the univariate and multivariate analyses.

\section{Diagnostic Value of US in Assessing the Feasibility of Complete Cytoreduction}

Recent studies $(24,25)$ have indicated that preoperative imaging techniques, such as CT and magnetic resonance imaging (MRI), could predict the resectability of PMP. Additionally, some US manifestations were significant prognostic factors for the resectability in PMP cases. The univariate analysis showed that omental cake was a poor prognostic factor for CC score. However, clear ascites, the appearance of liver scalloping, and abdominal masses were independent predictors of a higher CC score, which indicated a greater likelihood of incomplete cytoreduction. US showed lesions in the lesser omental bursa in the cases of gastric involvement, predicting a poorer CC score. Preoperative US evaluations of resectability helped to optimize the selection of patients who should undergo CRS, which could prevent additional unnecessary surgeries. These findings should be considered when explaining benefits and risks to patients before surgery.

\section{Predictive Value of US in Survival Rates}

The present study indicated that porta hepatis and the mesenterium were involved in the high-grade group. However, the multivariate analysis showed that only these two US features were highly correlated with the resectability of the lesions as well as the survival rates. This finding was comparable with that of a previous literature (26). The porta hepatis had complex anatomical relationships with the hepatic artery, portal vein, common bile duct, gallbladder triangle, and other important anatomical structures. It was difficult to clear all the hepatoduodenal and hepatogastric ligament tumors which severely affected the outcome of surgery. It demonstrated that the presence of residual unresectable disease in porta hepatis may influence the prognosis (25) and might explain why the involvement of the porta hepatis predicted PMP outcomes. The evaluation of porta hepatis using two-dimensional US has previously been reported to be associated with the unresectability of PMP; however, to the best of our knowledge, no study has reported its predictive role for CC score and survival rates. The present study indicated that portal infiltration helped in the early determination of resectability and survival rates.

Multivariate analysis identified mesenteric involvement as an independent predictor of a poorer CC score and survival rates. It was impossible to achieve extensive surgical resection for optimal treatment owing to irresectable small-bowel involvement, which was consistent with the study reported by Mittal et al. (2). In the present study, high-grade pathology and not undergoing HIPEC were also identified as independent predictors of poorer survival rates according to the multivariate analysis, which was consistent with previous reports (27). A simple-to-use nomogram that included these independent factors was developed using our 
TABLE 5 | The Cox regression model with US characteristics.

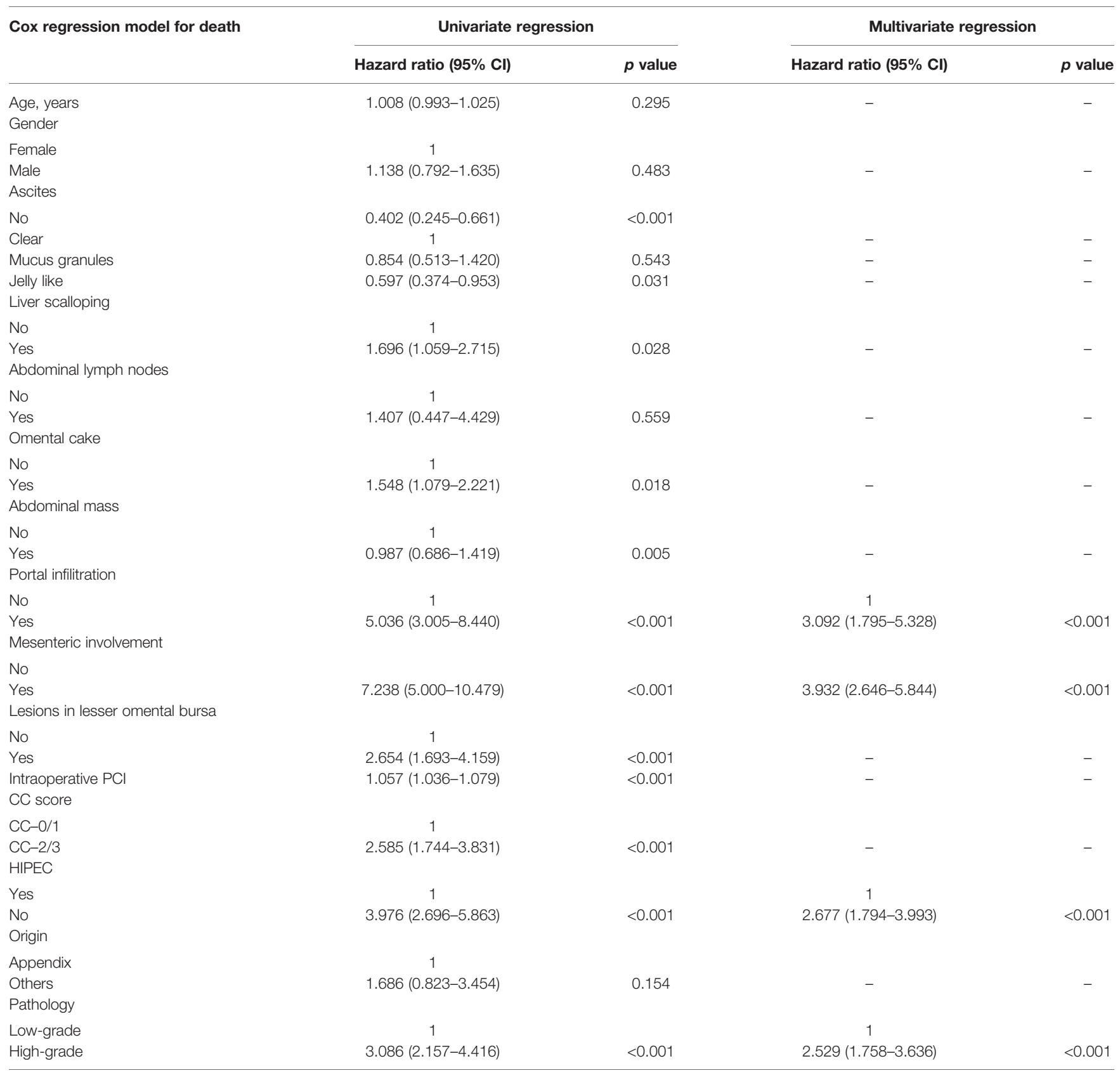

study data, which might be a useful visualization tool for predicting 1-, 2-, and 3-year survival probabilities for PMP in clinical practice.

When variables were examined using a univariate analysis, the CC score was associated with the survival rates. Maximal cytoreduction offered a greater chance of long-term survival. However, the multivariate analysis showed that the CC score was not a significant independent factor for survival outcomes. This was in contrast to the study reported by Chua et al. (28) and Ma et al. (29), which demonstrated that CC score was independently correlated with overall survival. The differences in patient factors and previous surgical or medical therapies between our results and their reports may contribute to the discrepant findings.

\section{Limitations}

This research had several limitations. First, although the PMP patient population was recruited from the core hospital for PMP surgery, our study was still a single-center study in a relatively small cohort. Secondly, although all US procedures and evaluations were performed by two doctors with 10 years of PMP examination experience, US is highly dependent on an individual skill level. This may reduce the repeatability of US 
results compared with that of the CT results to some extent. Finally, we had no data on urological or vascular involvement. We aim to acquire additional data for further study in the future.

\section{CONCLUSION}

Preoperative US has the potential value in predicting pathological grade, complete cytoreduction possibility, and the benefits of the surgery. Portal infiltration and mesenteric involvement are independent predictors of poorer survival rates. Moreover, a simple-to-use nomogram can effectively predict 1-, 2-, and 3-year survival of PMP patients, which may be considered for clinical utility.

\section{DATA AVAILABILITY STATEMENT}

The raw data supporting the conclusions of this article will be made available by the authors, without undue reservation.

\section{ETHICS STATEMENT}

The studies involving human participants were reviewed and approved by the Ethics Committee of Aerospace Center Hospital. The patients/participants provided their written informed consent to participate in this study. Written

\section{REFERENCES}

1. Carr NJ, Bibeau F, Bradley RF, Dartigues P, Feakins RM, Geisinger KR, et al. The Histopathological Classification, Diagnosis And Differential Diagnosis of Mucinous Appendiceal Neoplasms, Appendiceal Adenocarcinomas and Pseudomyxoma Peritonei. Histopathology (2017) 71:847-58. doi: 10.1111/his.13324

2. Mittal R, Chandramohan A, Moran B. Pseudomyxoma Peritonei: Natural History and Treatment. Int J Hyperthermia (2017) 33:511-9. doi: 10.1080/ 02656736.2017.1310938

3. Pillai K, Akhter J, Mekkawy A, Chua TC, Morris DL. Physical and Chemical Characteristics Of Mucin Secreted By Pseudomyxoma Peritonei (PMP). Int J Med Sci (2017) 14:18-28. doi: 10.7150/ijms.16422

4. Erickson LA, Rivera M. Mucinous Neoplasm of the Appendix and Pseudomyxoma Peritonei. Mayo Clin Proc (2018) 93:267-8. doi: 10.1016/ j.mayocp.2017.12.013

5. Morera-Ocon FJ, Navarro-Campoy C. History of Pseudomyxoma Peritonei From its Origin to the First Decades Of The Twenty-First Century. World J Gastrointest Surg (2019) 11:358-64. doi: 10.4240/wjgs.v11.i9.358

6. Bartoş A, Bartoş D, Raluca S, Mitre C, Hadade A, Iancu I, et al. Cytoreductive Surgery and Hyperthermic Intraperitoneal Chemotherapy for the Treatment of Peritoneal Carcinomatosis: Our Initial Experience. Chirurgia (Bucur) (2019) 114:222-33. doi: 10.21614/chirurgia.114.2.222

7. Benhaim L, Faron M, Gelli M, Sourrouille I, Honoré C, Delhorme JB, et al. Survival After Complete Cytoreductive Surgery and HIPEC for Extensive Pseudomyxoma Peritonei. Surg Oncol (2019) 29:78-83. doi: 10.1016/j.suronc.2019.03.004

8. Lee JL, Kim M, Kim J, Kim CW, Ha YJ, Kim SY, et al. Evaluation of the Significance Of Pseudomyxoma Peritonei Patients Based on the Peritoneal informed consent was obtained from the individual(s) for the publication of any potentially identifiable images or data included in this article.

\section{AUTHOR CONTRIBUTIONS}

All authors contributed to the study conception and design. Material preparation was performed by XH, NZ, and HX. Data collection and analysis were performed by $\mathrm{XH}, \mathrm{NZ}$, and QZ. The first draft of the manuscript was written by XH and LL. QZ and JG revised the manuscript critically for important intellectual content, and all authors commented on previous versions of the manuscript. All authors contributed to the article and approved the submitted version.

\section{FUNDING}

This work was supported by the Gold-Bridge Funds for Beijing (grant number ZZ21054), Aerospace Center Hospital Foundation (grant number YN201710), Beijing Talents Fund (grant number 2018000021469G198), and Natural Science Foundation of Beijing Municipal (grant number 7204249).

\section{ACKNOWLEDGMENTS}

The authors thank AiMi Academic Services and The Charlesworth Group for English language editing and review services.

Surface Oncology Group International (PSOGI) Classification. Asian J Surg S (2021) 44:848-53. doi: 10.1016/j.asjsur.2021.01.012

9. Lu YY, Qi CH, Li F, Hou F, Hao ZH, Pang SJ, et al. Diagnostic Value of Cytopathology in Pseudomyxoma Peritonei. J Diag Pathol (2017) 24:689-92. doi: 10.3969/j.issn.1007-8096.2017.09.012

10. Li ZS, Li Q. The Latest 2010 WHO Classification of Tumors of Digestive System. Zhonghua Bing Li Xue Za Zhi (2011) 40:351-4. doi: 10.3760/ cma.j.issn.0529-5807.2011.05.019

11. Fléjou JF. WHO Classification of Digestive Tumors: The Fourth Edition. Ann Pathol (2011) 31:S27-31. doi: 10.1016/j.annpat.2011.08.001

12. Valasek MA, Pai RK. An Update on the Diagnosis, Grading, and Staging of Appendiceal Mucinous Neoplasms. Adv Anat Pathol (2018) 25:38-60. doi: 10.1097/PAP.0000000000000178

13. Dohan A, Hoeffel C, Soyer P, Jannot AS, Valette PJ, Thivolet A, et al. Evaluation of the Peritoneal Carcinomatosis Index With CT and MRI. Br J Surg (2017) 104:1244-9. doi: 10.1002/bjs.10527

14. Menassel B, Duclos A, Passot G, Dohan A, Payet C, Isaac S, et al. Preoperative CT and MRI Prediction of Non-Resectability in Patients Treated for Pseudomyxoma Peritonei From Mucinous Appendiceal Neoplasms. Eur J Surg Oncol (2016) 42:558-66. doi: 10.1016/j.ejso.2016.01.005

15. Krause J, Bergman A, Graf W, Nilsson A, Mahteme H. Ultrasonography Findings and Tumour Quantification in Patients With Pseudomyxoma Peritonei. Eur J Radiol (2012) 81:648-51. doi: 10.1016/j.ejrad.2011.01.033

16. Liang L, Wang W, Zhou N, Guo J, Lu Y, Xu H, et al. Value of Preoperative Ultrasound in Evaluating the Peritoneal Cancer Index of Pseudomyxoma Peritonei. World J Surg Oncol (2019) 17:192. doi: 10.1186/s12957-0191730-5 
17. Ramaswamy V. Pathology of Mucinous Appendiceal Tumors and Pseudomyxoma Peritonei. Indian J Surg Oncol (2016) 7:258-67. doi: 10.1007/s13193-016-0516-2

18. Amini A, Masoumi-Moghaddam S, Ehteda A, Morris DL. Secreted Mucins in Pseudomyxoma Peritonei: Pathophysiological Significance and Potential Therapeutic Prospects. Orphanet J Rare Dis (2014) 9:71. doi: 10.1186/17501172-9-71

19. Fonseca C, Carvalho S, Cunha TM, Gil RT, Abecasis N. The Many Faces of Pseudomyxoma Peritonei: A Radiological Review Based on 30 Cases. Radiol Bras (2019) 52:372-7. doi: 10.1590/0100-3984.2019.0044

20. Fonseca EKUN, Martins AN, Tridente CF, Yamauchi FI, Baroni RH. Liver Scalloping in Pseudomyxoma Peritonei. Abdom Radiol (NY) (2017) 42:20034. doi: 10.1007/s00261-017-1092-9

21. Garach NR, Kusamura S, Guaglio M, Bartolini V, Deraco M, Baratti D. Comparative Study of Mucinous and Non-Mucinous Appendiceal Neoplasms With Peritoneal Dissemination Treated by Cyoreductive Surgery and Hyperthermic Intraperitoneal Chemotherapy (HIPEC). Eur J Surg Oncol (2020) 47:1132-9. doi: 10.1016/j.ejso.2020.08.017

22. Li Y, Duo A, Tang J, Wang L, Wang J, Yu D. Role of Preoperative Sonography in the Diagnosis and Pathologic Staging of Pseudomyxoma Peritonei. J Ultrasound Med (2013) 32:1565-70. doi: 10.7863/ultra.32.9.1565

23. Wasnik AP, Maturen KE, Kaza RK, Al-Hawary MM, Francis IR. Primary and Secondary Disease of the Peritoneum and Mesentery: Review of Anatomy and Imaging Features. Abdom Imaging (2015) 40:626-42. doi: 10.1007/s00261-014-0232-8

24. Low RN, Barone RM, Rousset P. Peritoneal MRI in Patients Undergoing Cytoreductive Surgery and HIPEC: History, Clinical Applications, and Implementation. Eur J Surg Oncol (2021) 47:65-74. doi: 10.1016/j. ejso.2019.02.030

25. Sinukumar S, Mehta S, As R, Damodaran D, Ray M, Zaveri S, et al. Analysis of Clinical Outcomes of Pseudomyxoma Peritonei From Appendicular Origin Following Cytoreductive Surgery and Hyperthermic Intraperitoneal Chemotherapy-A Retrospective Study From INDEPSO. Indian J Surg Oncol (2019) 10:65-70. doi: 10.1007/s13193-018-00870-w
26. Bouquot M, Dohan A, Gayat E, Barat M, Glehen O, Pocard M, et al. Prediction of Resectability in Pseudomyxoma Peritonei With a New CT Score. Ann Surg Oncol (2018) 25:694-701. doi: 10.1245/s10434-017-6275-7

27. Narasimhan V, Wilson K, Britto M, Warrier S, Lynch AC, Michael M, et al. Outcomes Following Cytoreduction and HIPEC for Pseudomyxoma Peritonei: 10-Year Experience. J Gastrointest Surg (2020) 24:899-906. doi: 10.1007/s11605-019-04239-4

28. Chua TC, Moran BJ, Sugarbaker PH, Levine EA, Glehen O, Gilly FN, et al. Early- and Long-Term Outcome Data of Patients With Pseudomyxoma Peritonei From Appendiceal Origin Treated by a Strategy of Cytoreductive Surgery and Hyperthermic Intraperitoneal Chemotherapy. J Clin Oncol (2012) 30:2449-56. doi: 10.1200/JCO.2011.39.7166

29. Ma R, Wang B, Zhai X, Lu Y, Xu H. Management and Prognostic Prediction of Appendiceal Mucinous Adenocarcinoma With Peritoneal Metastasis: A Single Center Study in China. BMC Cancer (2020) 20:280. doi: 10.1186/ s12885-020-06787-4

Conflict of Interest: The authors declare that the research was conducted in the absence of any commercial or financial relationships that could be construed as a potential conflict of interest.

Publisher's Note: All claims expressed in this article are solely those of the authors and do not necessarily represent those of their affiliated organizations, or those of the publisher, the editors and the reviewers. Any product that may be evaluated in this article, or claim that may be made by its manufacturer, is not guaranteed or endorsed by the publisher.

Copyright (c) 2021 Liang, Han, Zhou, Xu, Guo and Zhang. This is an open-access article distributed under the terms of the Creative Commons Attribution License (CC BY). The use, distribution or reproduction in other forums is permitted, provided the original author(s) and the copyright owner(s) are credited and that the original publication in this journal is cited, in accordance with accepted academic practice. No use, distribution or reproduction is permitted which does not comply with these terms. 